\title{
Bacteraemia, antimicrobial susceptibility and treatment among Campylobacter-associated hospitalisations in the Australian Capital Territory: a review
}

\author{
Cameron R. M. Moffatt ${ }^{1 *} \mathbb{D}$, Karina J. Kennedy ${ }^{2}$, Ben O'Neill ${ }^{1}$, Linda Selvey ${ }^{3}$ and Martyn D. Kirk
}

\begin{abstract}
Background: Campylobacter spp. cause mostly self-limiting enterocolitis, although a significant proportion of cases require hospitalisation highlighting potential for severe disease. Among people admitted, blood culture specimens are frequently collected and antibiotic treatment is initiated. We sought to understand clinical and host factors associated with bacteraemia, antibiotic treatment and isolate non-susceptibility among Campylobacter-associated hospitalisations.

Methods: Using linked hospital microbiology and administrative data we identified and reviewed Campylobacterassociated hospitalisations between 2004 and 2013. We calculated population-level incidence for Campylobacter bacteraemia and used logistic regression to examine factors associated with bacteraemia, antibiotic treatment and isolate non-susceptibility among Campylobacter-associated hospitalisations.

Results: Among 685 Campylobacter-associated hospitalisations, we identified 25 admissions for bacteraemia, an estimated incidence of 0.71 cases per 100,000 population per year. Around half of hospitalisations (333/685) had blood culturing performed. Factors associated with bacteraemia included underlying liver disease (aOR 48.89, 95\% Cl $7.03-340.22, p<0.001$ ), Haematology unit admission (aOR 14.67, 95\% Cl 2.99-72.07, $p=0.001$ ) and age 70-79 years (aOR 4.93, 95\% Cl 1.57-15.49). Approximately one-third (219/685) of admissions received antibiotics with treatment rates increasing significantly over time $(p<0.05)$. Factors associated with antibiotic treatment included Gastroenterology unit admission (aOR 3.75, 95\% Cl 1.95-7.20, p<0.001), having blood cultures taken (aOR 2.76, 95\% Cl 1.79-4.26, $p<0.001$ ) and age $40-49$ years (aOR $2.34,95 \% \mathrm{Cl} 1.14-4.79, p=0.02$ ). Non-susceptibility of isolates to standard antimicrobials increased significantly over time $(p=0.01)$ and was associated with overseas travel $(a O R 11.8095 \% \mathrm{Cl}$ $3.18-43.83, p<0.001$ ) and negatively associated with tachycardia (aOR $0.48,95 \% \mathrm{Cl} 0.26-0.88, p=0.02$ ), suggesting a healthy traveller effect.
\end{abstract}

\footnotetext{
*Correspondence: cameron.moffatt@anu.edu.au

${ }^{1}$ National Centre for Epidemiology and Population Health, Research

School of Population Health, Australian National University,

2602 Canberra, ACT, Australia

Full list of author information is available at the end of the article
}

(C) The Author(s) 2021. Open Access This article is licensed under a Creative Commons Attribution 4.0 International License, which permits use, sharing, adaptation, distribution and reproduction in any medium or format, as long as you give appropriate credit to the original author(s) and the source, provide a link to the Creative Commons licence, and indicate if changes were made. The images or other third party material in this article are included in the article's Creative Commons licence, unless indicated otherwise in a credit line to the material. If material is not included in the article's Creative Commons licence and your intended use is not permitted by statutory regulation or exceeds the permitted use, you will need to obtain permission directly from the copyright holder. To view a copy of this licence, visit http://creativecommons.org/licenses/by/4.0/. The Creative Commons Public Domain Dedication waiver (http://creativeco mmons.org/publicdomain/zero/1.0/) applies to the data made available in this article, unless otherwise stated in a credit line to the data. 
Conclusions: Campylobacter infections result in considerable hospital burden. Among those admitted to hospital, an interplay of factors involving clinical presentation, presence of underlying comorbidities, complications and increasing age influence how a case is investigated and managed.

Keywords: Campylobacter infections, Hospitalisation, Bacteraemia, Incidence, Antimicrobial susceptibility, Antimicrobial therapy, Comorbidity, Elderly

\section{Background}

Campylobacter spp. are internationally significant as a cause of infectious diarrhoeal disease [1]. Most cases of infectious diarrhoea, including those caused by Campylobacter spp., are self-limiting, with management focused on maintenance of hydration via fluid repletion [2]. However, for persons hospitalised with Campylobacter infection, clinical thresholds for exclusion of bacteraemia and the consideration of antimicrobial therapy differ due to symptom severity, risk of complications or the exacerbation of underlying co-morbidities [2].

While bacteraemia is an uncommon complication of campylobacteriosis, testing and diagnosis typically occurs when a person is hospitalised [3]. Within a hospital setting, clinical tolerances and the challenge of predicting bacteraemia among febrile admissions with an enteric focus are important considerations [4]. Further, the likelihood of clinicians commencing antibiotic therapy may also increase among hospitalised cases, highlighting the importance of judicial prescribing and understanding of isolate susceptibility patterns to ensure viable treatment options remain [5].

We describe rates of bacteraemia, antimicrobial susceptibility and treatment among a cohort of Campylobacter-associated hospitalisations. We also examined clinical and host factors associated with the diagnosis of blood stream infections (BSI), isolate non-susceptibility and antibiotic treatment of hospitalised cases.

\section{Methods}

\section{Background and data}

This study forms part of a retrospective review of Campylobacter-associated hospital admissions in the Australian Capital Territory (ACT) between January 2004 and December 2013. A Campylobacter-associated hospital admission was defined as any episode of care for an admitted patient that was clinically and temporally linked to a Campylobacter isolate derived from the same patient. Full details of the setting, data sources, data collection and linkage are described elsewhere [6]. In summary, they include hospital generated admission data, hospital microbiology data and clinical data obtained via individual medical record review. Hospital admission details were obtained via a data extract that included patient demographics, admission and discharge dates and admission unit details. Record inclusion was determined by an inpatient admission having been assigned an International Classification of Diseases (ICD) diagnosis code 'A045-Campylobacter enteritis'. We also obtained a separate microbiology data extract detailing inpatient isolations of Campylobacter spp., including specimen type, collection dates and antimicrobial susceptibility data. All laboratory diagnoses of Campylobacter infection were made via culture; speciation was not routinely performed on hospital isolates prior to 2013. Susceptibilities to ciprofloxacin, nalidixic acid and erythromycin were assessed using disk diffusion and according to Clinical and Laboratory Standards Institute breakpoints [7]. Intermediate and resistant isolates were grouped as non-susceptible to aid analysis. Due to the potential for multiple specimens being collected during an individual admission (or across multiple related admissions), we reported antimicrobial susceptibility using the earliest available data. Microbiology results were then linked to both admissions with and without ICD code 'A045'. We undertook a review of medical records to collect additional details on illness presentation, associated complications, patient co-morbidities (as per the Charlson Co-morbidity Index) [8] and antibiotic treatment and prescribing details (e.g., antibiotic type, dosage, frequency and administration route). For antibiotic treatment, we defined a total daily dose as the dosage in milligrams $(\mathrm{mg})$ multiplied by the frequency of administration per day, with the product expressed in milligrams per day.

\section{Statistical analysis}

We calculated bacteraemia incidence per 100,000 persons using the ACT's mid-year estimated resident population for each year between 2004 and 2013 [9]. We used non-parametric methods, including median tests, to analyse non-normally distributed variables such as age. We used Pearson's chi-squared test and Fisher's exact tests to assess simple statistical associations between key outcome and independent variables of interest, while trends in proportions were assessed using chi-squared tests. Preliminary analyses included the estimation of relative risks (RRs), 95\% confidence intervals (CIs) and p-values to assess potential predictors of blood culturing, antimicrobial susceptibility and antibiotic treatment. Variables examined included age, sex, country of birth, previous 
Campylobacter-associated hospitalisation, overseas travel history, signs and symptoms, admission unit, comorbidities and the presence of key signs of infection. Using a stepwise additive approach, with the most significant variables being added first, we constructed separate logistic regression models to identify predictors associated with (i) collection of blood specimens for culture, (ii) positive blood isolates, (iii) non-susceptibility to ciprofloxacin, (iv) any antimicrobial non-susceptibility and (vii) antibiotic treatment during hospitalisation. Erythromycin non-susceptibility was not assessed due to the limited number of observations. Clinical relevance and statistical evidence were used to assist with variable selection for multivariable analysis. The significance level for removal from the models was set at $\mathrm{p} \leq 0.05$. We used likelihoodratio tests to assess the explanatory power of the models, with the variable expressing the largest $p$-value being removed. Final results were expressed as adjusted odds ratios (aOR), with accompanying 95\% confidence intervals and $p$-values. We used Hosmer-Lemeshow tests to assess the goodness-of-fit for each model. All statistical analyses were performed using Stata v.14 (StataCorp, USA).

\section{Results}

\section{Campylobacter bacteraemia}

Out of 685 admissions, 333 (49\%) had blood drawn for culture and $25(7.5 \%)$ of these tested positive (Fig. 1). Speciation was performed on 21 (84\%) of these case isolates with 15 (71.4\%) cases of C. jejuni, 5 (23.8\%) cases of C. coli and a single (4.0\%) case of C. lari bacteraemia. Amongst the 25 admissions with bacteraemia, 15 were male $(60 \%)$ and 10 were female (40\%), while the median age was 59.5 years (range 12 to 90 years). Amongst 308 negative cases there were 177 males $(57.5 \%)$ and 131 females $(42.5 \%)$, with a median age of 38.8 years (age range $<1.0$ to 92 years). This age difference was significant (median test $\chi^{2}=5.30, p=0.02$ ) but no evidence of a difference in the sex composition was observed $\left(\chi^{2}=0.06, p=0.81\right)$. The age and sex of admissions in relation to blood culture status is shown in Fig. 2. We also compared those who had blood drawn for culture (whether positive or negative) with those that did not to assess demographic differences. We saw no evidence of a difference in median age (median test $\chi^{2}=0.18$, $p=0.68$ ) but did observe males to be more likely to have blood drawn $\left(\chi^{2}=11.17, p=0.001\right)$. We observed no difference in the proportion of admissions with documented comorbidities who had blood specimens collected for culture when compared to admission without comorbidities who had blood taken for culture $\left(\chi^{2}=1.42, p=0.23\right)$. Bacteraemia generally occurred in the context of antecedent diarrhoeal illness,

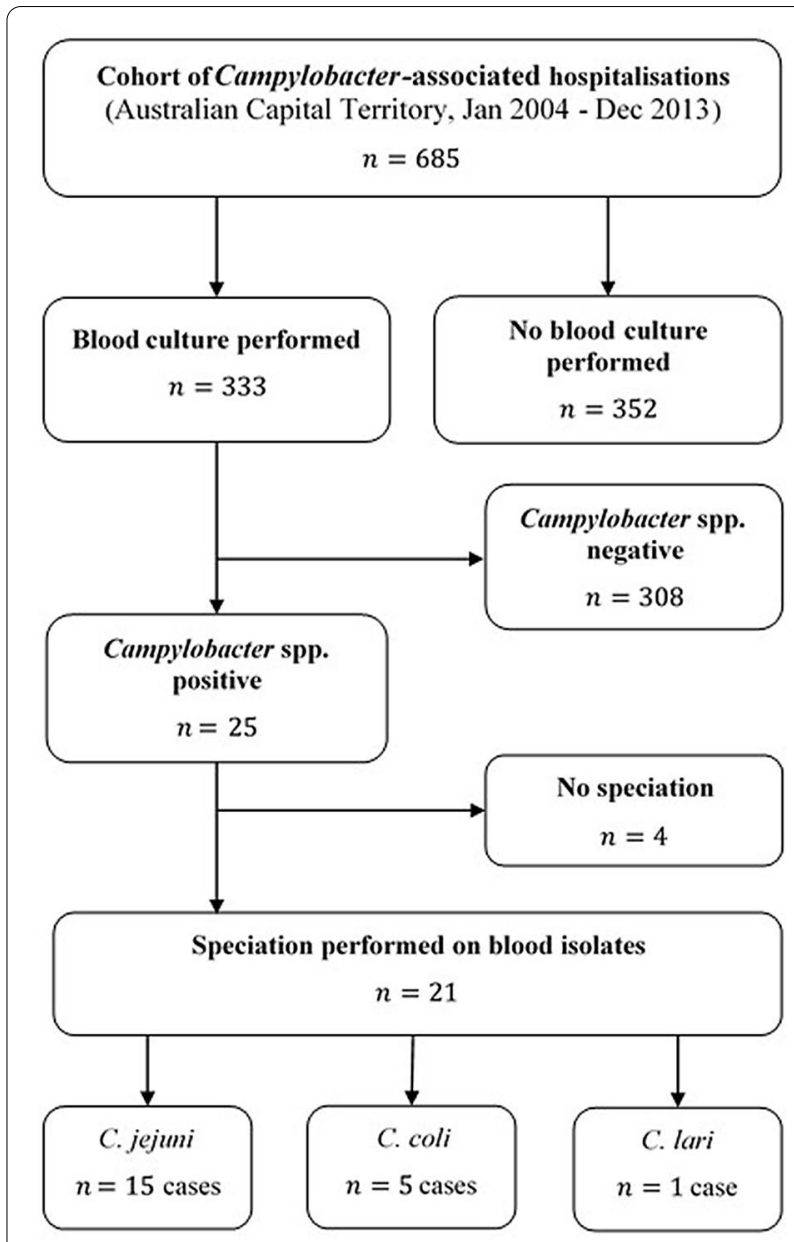

Fig. 1 Flow diagram for blood culture collection and subsequent Campylobacter spp. bacteraemia among a cohort of Campylobacter-associated hospitalisations

although two admissions involved primary bacteraemia (i.e. without diarrhoea) in patients with haematological malignancies. Comorbidities, while common, were not a characteristic feature of cases hospitalised with bacteraemia as shown in Table 1. No deaths were identified among cases with bacteraemia.

During the period 2004 to 2013, the mean incidence of Campylobacter bacteraemia in the host population was 0.71 cases per 100,000 population per year $(95 \%$ CI $0.48-1.05$ per 100,000 population) (Fig. 3 ). We did not observe temporal trends in the proportion of Campylobacter-associated hospitalisations undergoing blood collection for culture or in the proportion of positive blood cultures among Campylobacter-associated hospitalisations.

Factors associated with the collection of blood samples for culture and subsequent isolation of Campylobacter spp. are shown in Tables 2 and 3. 


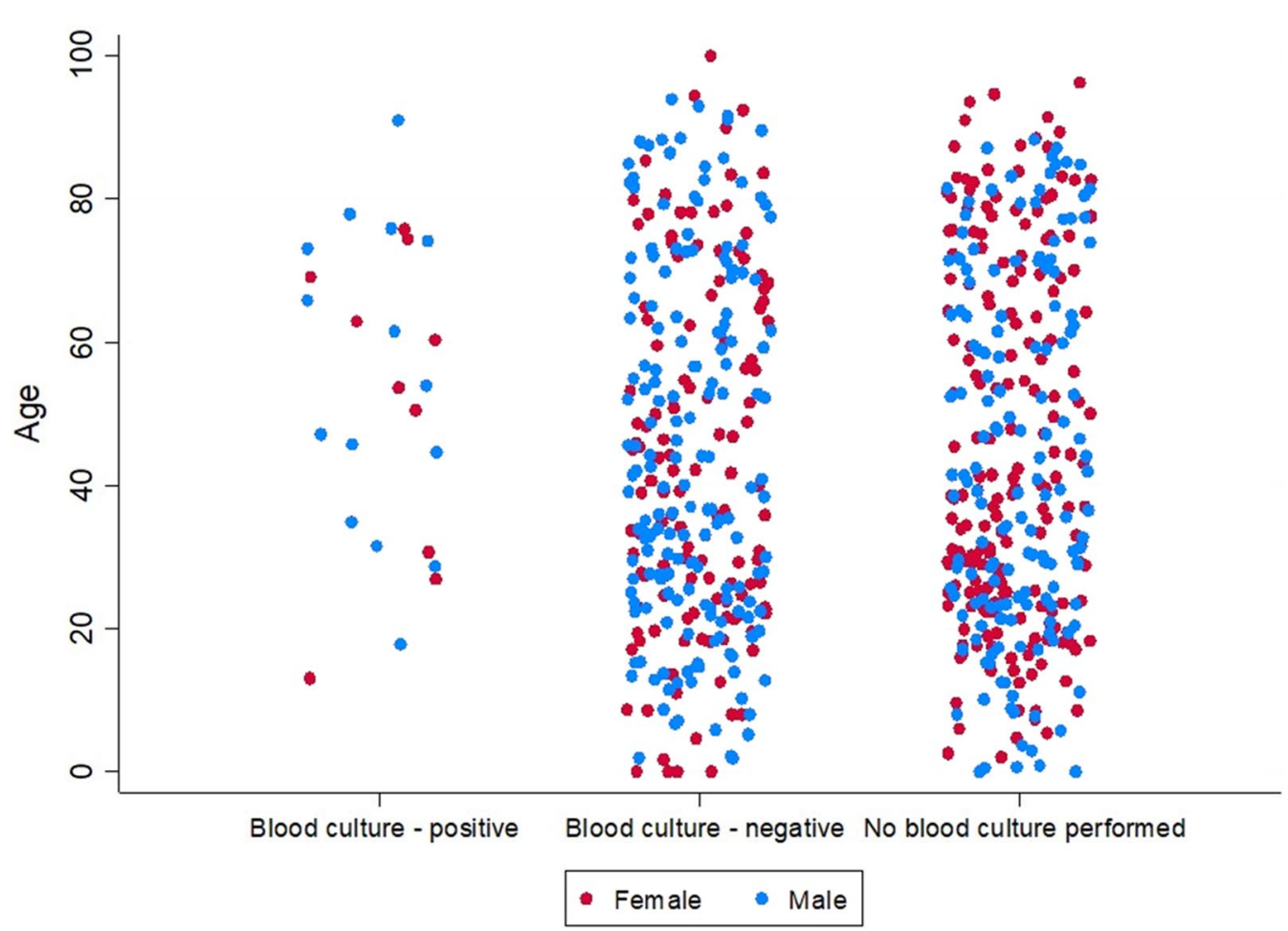

Fig. 2 Jitter plot of age and sex by blood culture status among Campylobacter-associated hospitalisations

\section{Antimicrobial susceptibility}

We identified 548 Campylobacter-associated admissions with a primary isolation of Campylobacter spp. and where subsequent antimicrobial susceptibility testing was performed. For 526 (96\%) of these cases there was available data for three standard antimicrobials, ciprofloxacin, nalidixic acid and erythromycin. Of the remainder, nalidixic acid data was unavailable for 21 isolates, with erythromycin susceptibility unavailable for a single isolate.

During the study period, $13 \%$ (70/548) of primary isolates exhibited non-susceptibility to at least one standard antimicrobial. The proportion of non-susceptible isolates ranged from $\leq 5.0 \%$ in $2004 / 05$ to $>20.0 \%$ in $2012 / 13$, with this increase being significant $\left(\chi^{2}=6.12, p=0.01\right)$ (Fig. 4). Among the individual antimicrobials, nalidixic acid non-susceptibility was reported for 9\% (49/527) of tested isolates, $7 \%$ (40/548) for ciprofloxacin and $4 \%$ $(21 / 547)$ for erythromycin.

Figure 4 shows that lower rates of erythromycin nonsusceptibility were observed while significant increases in both ciprofloxacin $\left(\chi^{2}=16.51, p<0.001\right)$ and nalidixic acid non-susceptibility occurred during the study period $\left(x^{2}=10.85, p=0.001\right)$. Factors associated with antimicrobial non-susceptibility among Campylobacter-associated hospitalisations are shown in Tables 4 and 5.

\section{Antibiotic treatment}

Antimicrobial treatment was provided for 32\% (219/685) of Campylobacter-associated hospitalisations. Those receiving treatment were observed to be significantly older (median 48.5 years, range 8 years to 92 years) than admissions where antibiotics were not administered (median 34.8 years, range $<1$ years to 91 years, $\chi^{2}=26.92$, $p<0.001)$. No sex-based differences were observed. Treatment associated with bacteraemia is described in Table 1.

Second generation fluoroquinolones were used in $79 \%(172 / 219)$ of treated admissions. Ciprofloxacin was administered to $82.0 \%(141 / 172)$, with the remainder receiving norfloxacin. Those receiving ciprofloxacin were younger (median 47.2 years, minimum 12 years, maximum 90 years) compared with those receiving norfloxacin (median age 64.3 years, minimum 19 years to maximum 92 years), but this difference was not significant. For admissions treated with ciprofloxacin, 91.4\% $(129 / 141)$ received treatment orally, with a median total daily dose of $1000 \mathrm{mg}$. For those receiving parenteral ciprofloxacin $(n=12)$, the median total daily dose was $800 \mathrm{mg}$. The median total daily dosage for oral norfloxa$\operatorname{cin}(n=31)$ was $800 \mathrm{mg}$.

Macrolides (azithromycin or erythromycin) were administered to $21.0 \%(46 / 219)$ of admissions, with 
Table 1 Characteristics of cases hospitalised with Campylobacter bacteraemia, 2004 to 2013

\begin{tabular}{|c|c|c|c|c|c|c|}
\hline Year (case) & Age range/sex & Species & $\begin{array}{l}\text { Bacteraemia- } \\
\text { source }\end{array}$ & $\begin{array}{l}\text { Antimicrobial } \\
\text { susceptibility }\end{array}$ & $\begin{array}{l}\text { Antimicrobial } \\
\text { treatment }\end{array}$ & $\begin{array}{l}\text { Significant medical } \\
\text { history and risk factors }\end{array}$ \\
\hline 2004 & $80+M$ & C.jejuni & Enteric_-secondary & Fully sensitive & $\mathrm{Nil}$ & Age, nil other significant \\
\hline $2005(a)$ & $50-59 F$ & Campylobacter sp. & Enteric-secondary & Fully sensitive & $\begin{array}{l}\text { PO ciprofloxacin } \\
500 \text { mg bd }\end{array}$ & $\begin{array}{l}\text { Acute myeloid leukae- } \\
\text { mia }\end{array}$ \\
\hline $2005(b)$ & $60-69 F$ & Campylobacter sp. & Enteric-secondary & Fully sensitive & $\mathrm{Nil}$ & Nil significant \\
\hline $2005(c)$ & $40-49 F$ & C.jejuni & Enteric_-secondary & Fully sensitive & $\begin{array}{l}\text { PO ciprofloxacin } \\
500 \text { mg bd }\end{array}$ & $\begin{array}{l}\text { IV drug use, PUD on } \\
\text { omeprazole }\end{array}$ \\
\hline $2006(a)$ & $40-49 M$ & C.jejuni & Enteric - secondary & Fully sensitive & $\begin{array}{l}\text { PO ciprofloxacin } \\
500 \text { mg bd }\end{array}$ & Untreated Stage III HIV \\
\hline $2008(a)$ & $20-29 \mathrm{M}$ & Campylobacter sp. & Enteric_-secondary & Fully sensitive & $\begin{array}{l}\text { IV azithromycin } \\
500 \text { mg qd }\end{array}$ & Nil significant \\
\hline $2008(b)$ & $60-69 M$ & C. coli & Primary bacteraemia & $\begin{array}{l}\text { Ciprofloxacin- Resist- } \\
\text { ant } \\
\text { Erythromycin- Resist- } \\
\text { ant }\end{array}$ & Nil & Lymphocytic lymphoma \\
\hline 2009 & $30-39 F$ & C.jejuni & Enteric-secondary & Fully sensitive & $\begin{array}{l}\text { PO ciprofloxacin } \\
250 \text { mg bd }\end{array}$ & $\begin{array}{l}\text { History of renal trans- } \\
\text { plant secondary to lgA } \\
\text { nephropathy }\end{array}$ \\
\hline $2010(a)$ & $60-69 M$ & Campylobacter sp. & Enteric_-secondary & Fully sensitive & $\begin{array}{l}\text { PO ciprofloxacin } \\
500 \text { mg bd }\end{array}$ & $\begin{array}{l}\text { Bowel carcinoma, cur- } \\
\text { rent chemotherapy }\end{array}$ \\
\hline $2010(b)$ & $30-39 M$ & C. coli & Enteric_-secondary & Fully sensitive & $\begin{array}{l}\text { PO ciprofloxacin } \\
500 \text { mg bd }\end{array}$ & $\begin{array}{l}\text { Alcoholic liver disease } \\
\text { with portal hypoten- } \\
\text { sion and bleeding } \\
\text { varices }\end{array}$ \\
\hline $2010(c)$ & 70-79 F & C.jejuni & Enteric_-secondary & Fully sensitive & $\begin{array}{l}\text { PO ciprofloxacin } \\
500 \text { mg bd }\end{array}$ & T2DM \\
\hline $2010(d)$ & $10-19 F$ & C.jejuni & Enteric-secondary & Fully sensitive & $\begin{array}{l}\text { PO azithromycin } \\
500 \text { mg qd (upon } \\
\text { discharge) }\end{array}$ & Nil significant. \\
\hline $2010(e)$ & 70-79 F & C. coli & Enteric-secondary & Fully sensitive & $\begin{array}{l}\text { PO ciprofloxacin } \\
500 \text { mg bd }\end{array}$ & T2DM \\
\hline $2010(f)$ & $40-49 \mathrm{M}$ & C.jejuni & Enteric_-secondary & Fully sensitive & $\begin{array}{l}\text { PO Norfloxacin } 400 \text { mg } \\
\text { bd }\end{array}$ & $\begin{array}{l}\text { Irritable bowel syn- } \\
\text { drome }\end{array}$ \\
\hline $2011(a)$ & $60-69 M$ & C.lari & Enteric-secondary & $\begin{array}{l}\text { Ciprofloxacin- Resist- } \\
\text { ant }\end{array}$ & $\begin{array}{l}\text { PO Doxycycline } \\
100 \text { mg bd }\end{array}$ & $\begin{array}{l}\text { Alcoholic liver disease } \\
\text { with portal hypoten- } \\
\text { sion, recent intracer- } \\
\text { ebral bleed }\end{array}$ \\
\hline $2011(b)$ & $80+M$ & C.jejuni & Enteric-secondary & Fully sensitive & Nil prescribed & Age, nil other significant \\
\hline $2011(c)$ & $70-79 \mathrm{M}$ & C. coli & Enteric-secondary & Fully sensitive & $\begin{array}{l}\text { PO ciprofloxacin } \\
500 \text { mg bd }\end{array}$ & Asplenic \\
\hline $2012(a)$ & $40-49 M$ & C.jejuni & Enteric_-secondary & Fully sensitive & $\begin{array}{l}\text { IV ciprofloxacin } \\
500 \text { mg bd }\end{array}$ & $\begin{array}{l}\text { Multiple sclerosis, cur- } \\
\text { rent chemotherapy } \\
\text { pre-stem cell trans- } \\
\text { plantation, IDDM }\end{array}$ \\
\hline $2012(b)$ & $30-39 F$ & C.jejuni & Enteric-secondary & Fully sensitive & Nil & Pregnant 33/40K, IDDM \\
\hline $2012(c)$ & $70-79 \mathrm{M}$ & C.jejuni & Enteric_-secondary & $\begin{array}{l}\text { Ciprofloxacin- Resist- } \\
\text { ant } \\
\text { Nalidixic acid-Resist- } \\
\text { ant }\end{array}$ & $\mathrm{Nil}$ & $\begin{array}{l}\text { Diabetic neuropathy, } \\
\text { chronic renal failure }\end{array}$ \\
\hline $2012(d)$ & $60-69 F$ & C. coli & Enteric-secondary & $\begin{array}{l}\text { Ciprofloxacin- Resist- } \\
\text { ant } \\
\text { Nalidixic acid - Resist- } \\
\text { ant }\end{array}$ & Nil & Nil significant \\
\hline $2013(a)$ & $20-29 \mathrm{M}$ & C.jejuni & Enteric_-secondary & Fully sensitive & $\mathrm{Nil}$ & Nil significant \\
\hline $2013(b)$ & $20-29 M$ & C.jejuni & Primary bacteraemia & Fully sensitive & $\begin{array}{l}\text { PO Ciprofloxacin } \\
750 \text { mg bd (upon } \\
\text { discharge) }\end{array}$ & $\begin{array}{r}\text { B cell leukaemia, AVN } \\
\text { (on steroids), SIADH }\end{array}$ \\
\hline
\end{tabular}


Table 1 (continued)

\begin{tabular}{|c|c|c|c|c|c|c|}
\hline Year (case) & Age range/sex & Species & $\begin{array}{l}\text { Bacteraemia- } \\
\text { source }\end{array}$ & $\begin{array}{l}\text { Antimicrobial } \\
\text { susceptibility }\end{array}$ & $\begin{array}{l}\text { Antimicrobial } \\
\text { treatment }\end{array}$ & $\begin{array}{l}\text { Significant medical } \\
\text { history and risk factors }\end{array}$ \\
\hline 2013 (c) & $50-59 \mathrm{M}$ & C. jejuni & Enteric_-secondary & Fully sensitive & $\begin{array}{l}\text { PO ciprofloxacin } \\
500 \text { mg bd }\end{array}$ & $\begin{array}{l}\text { Liver failure with cirrho- } \\
\text { sis, portal hypotension } \\
\text { secondary to Hepatitis } \\
\text { C,T2DM, hypothy- } \\
\text { roidism. Awaiting } \\
\text { transplant. }\end{array}$ \\
\hline $2013(d)$ & 70-79 M & C. jejuni & Enteric_-secondary & Fully sensitive & $\mathrm{Nil}$ & Age, nil other significant \\
\hline
\end{tabular}

PO, per oral; IV, intravenous; PUD, peptic ulcer disease; HIV, human immunodeficiency virus; T2DM, type 2 diabetes mellitus; IDDM, insulin dependent diabetes mellitus; AVN, acute vascular necrosis; SIADH, syndrome of inappropriate antidiuretic hormone

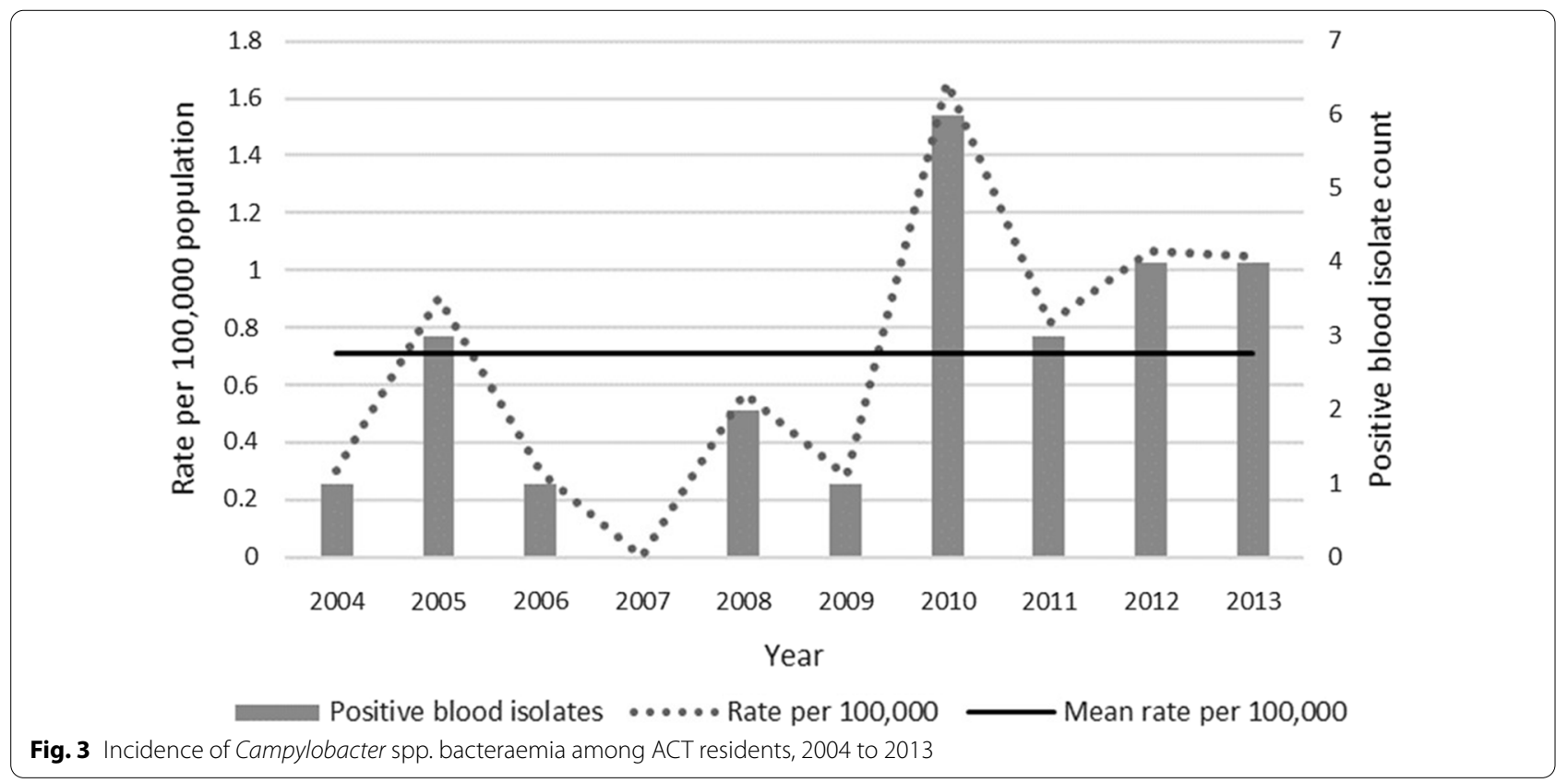

Table 2 Factors associated with collection of blood for culture among a cohort of Campylobacter-associated hospitalisations ( $\boldsymbol{n}=663)$

\begin{tabular}{lcccc}
\hline Predictor variable & Estimated adjusted Odds Ratio (aOR) & 95\% Confidence interval & $\boldsymbol{p}$-value \\
\hline Infectious Diseases Unit admission & 8.00 & $1.58-40.61$ & 0.01 \\
Febrile during admission $\left(\geq 38^{\circ} \mathrm{C}\right)$ & 21.30 & $13.76-32.96$ & $<.001$ \\
Tachycardia & 1.75 & $1.13-2.72$ & 0.01 \\
Moderate to severe renal disease & 2.87 & $1.26-6.55$ & 0.01 \\
$10-19$ years age group & 0.36 & $0.19-0.71$ & $<0.01$ \\
\hline
\end{tabular}

22 observations contained missing data

Hosmer and Lemeshow goodness of fit $\chi^{2}=2.70, p=0.61$

$63.0 \%(29 / 46)$ receiving azithromycin. No age or sex differences were observed between those receiving azithromycin versus erythromycin. For admissions receiving azithromycin, $72.4 \%(21 / 29)$ received treatment orally, with a median total daily dose for both oral and IV azithromycin of $500 \mathrm{mg}$. Oral administration was provided for $94.1 \%$ (16/17) of admissions receiving erythromycin, with a median total daily dose of $2000 \mathrm{mg}$. One patient received an initial total daily dose of $3200 \mathrm{mg}$ IV erythromycin, prior to this being reduced to a total daily dose of $1600 \mathrm{mg}$. For two admissions (involving the same patient), a tetracycline (doxycycline) was used to treat a C. lari bacteraemia and enterocolitis. 
Table 3 Factors associated with blood stream isolation of Campylobacter spp. among Campylobacter-associated hospitalisations $(n=333)$

\begin{tabular}{lllr}
\hline Predictor variable & Estimated adjusted Odds Ratio (aOR) & 95\% Confidence interval & $p$-value \\
\hline Moderate to severe liver disease & 48.89 & $7.03-340.22$ & $<0.001$ \\
Haematology Unit admission & 14.67 & $2.99-72.07$ & 0.001 \\
Age group 70-79 years & 4.93 & $1.57-15.49$ & $<0.01$ \\
Admission during summer months & 2.93 & $1.14-7.57$ & 0.03 \\
Indigenous Australian & 10.87 & $1.00-117.89$ & 0.05 \\
\hline
\end{tabular}

Hosmer and Lemshow goodness of fit $\chi^{2}=0.71, p=0.70$

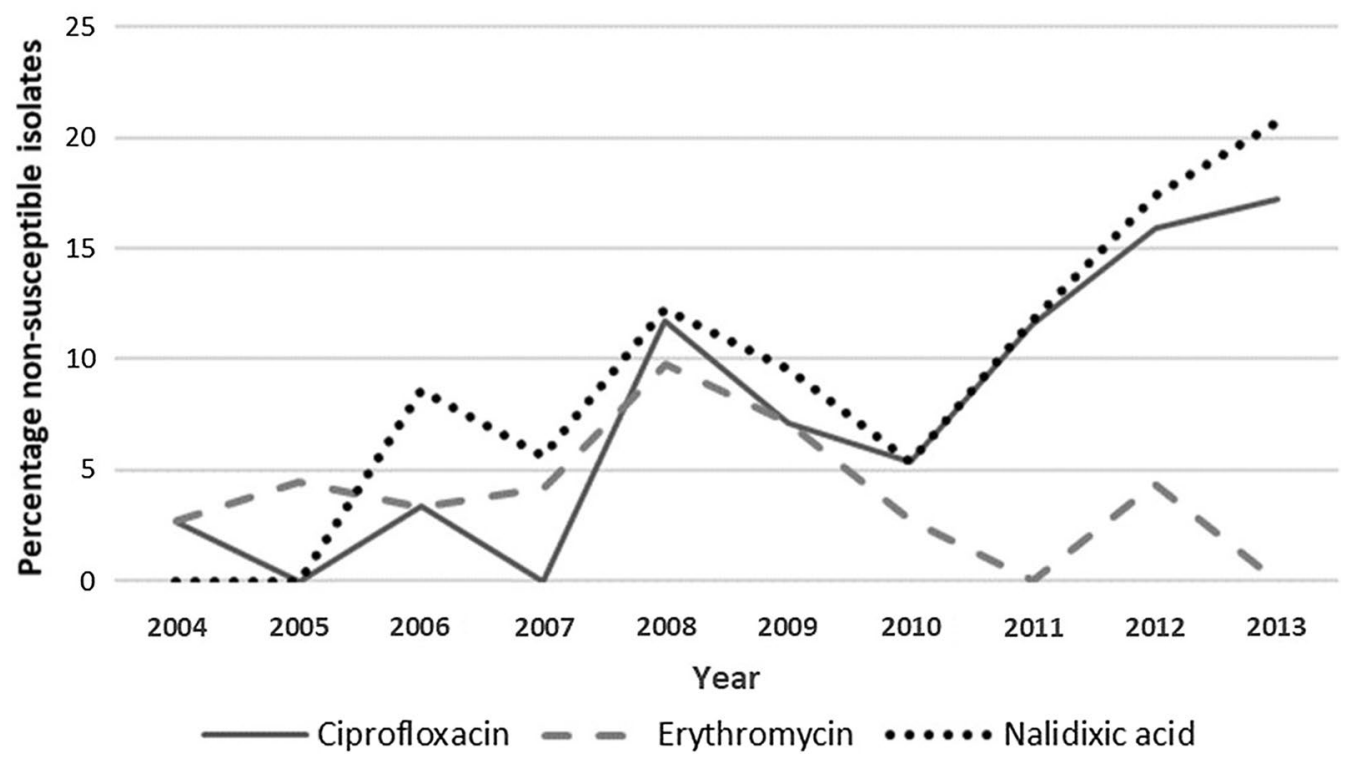

Fig. 4 Non-susceptibility to standard antimicrobials from Campylobacter isolates obtained during Campylobacter-associated hospitalisations, 2004 to 2013

Table 4 Factors associated with ciprofloxacin non-susceptible isolates among Campylobacter-associated hospitalisations $(n=411)$

\begin{tabular}{lcll}
\hline Predictor variable & $\begin{array}{l}\text { Estimated } \\
\text { adjusted Odds } \\
\text { Ratio (aOR) }\end{array}$ & $\begin{array}{l}\mathbf{9 5 \%} \\
\text { Confidence } \\
\text { interval }\end{array}$ & -value \\
\hline Bloody diarrhoea & 0.30 & $0.09-1.03$ & 0.06 \\
$\begin{array}{l}\text { Recent overseas travel } \\
\begin{array}{l}\text { Previous Campylobacter- } \\
\text { associated hospitalisa- } \\
\text { tion }\end{array}\end{array}$ & 15.53 & $2.86-84.18$ & 0.001 \\
\hline
\end{tabular}

Hosmer and Lemeshow goodness of fit $x^{2}=0.03, p=0.86$

A significant increase in the proportion of Campylobacter-associated hospitalisations being administered antimicrobials was observed over time $\left(\chi^{2}=4.37\right.$, $p=0.04)$, rising from $27 \%(14 / 52)$ in 2004 to $38 \%(26 / 68)$
Table 5 Factors associated with non-susceptibility to ciprofloxacin, nalidixic acid or erythromycin among a cohort of Campylobacter-associated hospitalisations $(n=548)$

\begin{tabular}{llll}
\hline Predictor variable & $\begin{array}{l}\text { Estimated } \\
\text { adjusted Odds } \\
\text { Ratio (aOR) }\end{array}$ & $\begin{array}{l}\mathbf{9 5 \%} \\
\text { Confidence } \\
\text { Interval }\end{array}$ & -value \\
\hline $\begin{array}{l}\text { Recent overseas travel } \\
\begin{array}{l}\text { Previous Campylobacter- } \\
\text { associated hospitalisa- } \\
\text { tion }\end{array}\end{array}$ & 11.80 & $3.18-43.83$ & $<0.001$ \\
$\begin{array}{l}\text { Tachycardia } \\
\text { Hosmer and Lemeshow goodness of fit } \mathrm{x}^{2}=0.01, p=0.92\end{array}$ & $2.65-110.07$ & $<0.01$ \\
\end{tabular}

of admissions in 2013. No difference in the proportion of admissions treated with either fluoroquinolones or macrolides was observed. Among admissions receiving macrolides a significant increase in the administration 
of azithromycin over erythromycin was observed $\left(x^{2}=16.31, p<0.001\right)$, most notably from 2011 onwards. No changes over time in the proportion of admissions treated with ciprofloxacin versus norfloxacin were observed. Factors associated with administration of antibiotics during Campylobacter-associated hospitalisations are shown in Table 6.

\section{Discussion}

We observed a high rate of bacteraemia in this study of Campylobacter-associated hospital admissions. Although blood cultures are not routinely performed for cases admitted with infectious gastroenteritis, we observed for Campylobacter-associated hospitalisations the presence of fever, pre-existing kidney disease or admission to particular subspecialty units increased the likelihood of blood being collected. Comorbidity and advanced age were both associated with a subsequent isolation from blood. One third of admissions received antibiotic treatment during the study period, with the proportion of those treated rising significantly over time. We also observed a temporal increase in the proportion of isolates exhibiting non-susceptibility to standard antimicrobials, notably fluoroquinolones. This is notable given the clinical and public health concern about development of antimicrobial resistance. Factors associated with ciprofloxacin resistance included recent overseas travel and previous hospitalisation for campylobacteriosis. This carries important clinical consequences, as the option to treat with antibiotics may carry greater importance

Table 6 Factors associated with antibiotic administration among a cohort of Campylobacter-associated hospitalisations $(n=607)$

\begin{tabular}{|c|c|c|c|}
\hline Predictor variable & $\begin{array}{l}\text { Estimated } \\
\text { adjusted Odds } \\
\text { Ratio (aOR) }\end{array}$ & $\begin{array}{l}95 \% \\
\text { Confidence } \\
\text { Interval }\end{array}$ & $p$-value \\
\hline Age $0-9$ years & 0.07 & $0.01-0.57$ & 0.01 \\
\hline Age 10-19 years & 0.44 & $0.20-0.97$ & 0.04 \\
\hline Age $40-49$ years & 2.34 & $1.14-4.79$ & 0.02 \\
\hline $\begin{array}{l}\text { Emergency Unit admis- } \\
\text { sion }\end{array}$ & 0.06 & $0.02-0.17$ & $<0.001$ \\
\hline $\begin{array}{l}\text { Gastroenterology admis- } \\
\text { sion }\end{array}$ & 3.75 & $1.95-7.20$ & $<0.001$ \\
\hline Gen. Medicine admission & 2.02 & $1.22-3.35$ & $<0.01$ \\
\hline $\begin{array}{l}\text { Infectious Diseases } \\
\text { admission }\end{array}$ & 2.58 & $1.03-6.44$ & 0.04 \\
\hline Vomiting & 1.70 & $1.11-2.61$ & 0.02 \\
\hline Electrolyte imbalance & 1.73 & $1.12-2.67$ & 0.01 \\
\hline $\begin{array}{l}\text { Blood specimen for } \\
\text { culture }\end{array}$ & 2.76 & $1.79-4.26$ & $<0.001$ \\
\hline
\end{tabular}

Hosmer and Lemeshow goodness of fit $x^{2} 9.23, p=0.32$ among hospitalised cases compared to non-hospitalised cases.

Campylobacteriosis most commonly presents as a self-limiting enterocolitis, with secondary bacteraemia a recognised, but relatively infrequent complication [10]. A number of studies have sought to determine the incidence of Campylobacter bacteraemia in high-income settings, estimating rates to be between 0.20 and 0.47 cases per 100,000 population [3, 11, 12]. A more recent Swedish study [13] has reported an incidence of 1.00 case per 100,000 population, with this linked to changes in automated blood culture collection systems. In our study we observed only 25 incident cases of bacteraemia, equating to a mean incidence of 0.71 cases per 100,000 population. While bacteraemia is relatively infrequent, our population rates appears high, likely reflecting high background incidence of campylobacteriosis in the ACT [14] and a lower threshold for testing among hospitalised cases.

Blood cultures are the gold standard for the diagnosis of BSIs [4]. In our study, patients who had a measured fever $\left(\geq 38{ }^{\circ} \mathrm{C}\right)$, underlying chronic kidney disease or who were admitted under the care of the Infectious Diseases Unit were more likely to have blood drawn for culture. Fever is a common prompt for blood cultures, with patients whose measured temperatures are $\geq 38{ }^{\circ} \mathrm{C}$ having increased likelihood of bacteraemia [15]. Similarly tachycardia, another vital sign, has also been used in clinical prediction rules for blood-stream infection [16]. Although comorbidities do not feature in BSI underlying kidney disease has also been shown as a risk factor for bloodstream infections in older patients [17]. Several consequences of kidney disease have been proposed to contribute to infection including malnutrition, chronic inflammation, retained uremic solutes, trace element deficiencies and metabolic abnormalities [18]. The finding that blood culture is more likely to be ordered by Infectious Diseases clinicians is unsurprising given the clinical focus of this subspecialty. Conversely, there was significant evidence that those aged between 10 and 19 years were less likely to have blood cultures performed. This finding likely reflects fewer hospitalisations being observed due to the low incidence of campylobacteriosis in this age grouping [14].

Predicting BSIs is challenging with numerous models developed for specific populations, settings and sources of infection [19]. The pre-test probability of bacteraemia will therefore vary considerably based upon the clinical context and source of infection [15]. Generally only $5-10 \%$ of blood cultures are positive, and of those positive results, between 30 and 50\% represent contaminants [19]. In our study we observed blood cultures to be routinely requested, with $49 \%$ of acute admissions having blood drawn and 7\% being positive for Campylobacter 
spp. These admissions involved both immunosuppressed and immunocompetent patients. The impacts of comorbid and immunosuppressive conditions on clinical variables used in predicting bacteraemia is less well understood [15], although associations with campylobacteriosis and invasive disease in immunosuppressed patients have been described [3, 20]. Transient bacteraemia may also be common among immunocompetent hosts with Campylobacter enterocolitis but is less frequently detected due to the bactericidal effect of human serum and reduced frequency of blood culture among acute enterocolitis patients [10], although nearly half of acute admissions in our study had blood culturing performed. Notably, similar proportions of acute admissions with and without comorbidities were observed to have blood drawn for culture suggesting in our study population that comorbidity exerted limited influence on decisions to request blood cultures. This is perhaps not surprising given prediction rules for BSI focus on clinical signs [15].

\section{Factors associated with a positive blood stream isolate (bacteraemia)}

The statistical association we observed between cases with positive blood cultures and the presence of underlying liver disease and haematological malignancy is in keeping with hospital-based studies showing higher proportions of these conditions among cases with Campylobacter bacteraemia [11, 21, 22]. In addition, advanced age was also statistically associated with detection of bacteraemia, a characteristic seen in larger population-based studies of campylobacteriosis [3]. Several studies also report seasonality with Campylobacter bacteraemia [23, 24]. Our results show hospitalisation during the southern hemisphere summer to be associated with bacteraemia, a finding that aligns with the seasonality of Campylobacter enteritis in Australia [14]. A further association with blood culture positivity was Indigenous status. This result was derived however from a small number of observations with, Indigenous Australians comprising 1\% of Campylobacter-associated hospitalisations in the ACT [6].

\section{Factors associated with non-susceptibility to antimicrobials}

We observed statistically significant evidence of an increase in the proportion of isolates exhibiting non-susceptibility to standard antimicrobials, with this increase driven primarily by non-susceptibility to fluoroquinolones (including nalidixic acid). In keeping with trends in comparable settings only low rates of non-susceptibility to macrolides were observed [25, 26]. Internationally, fluoroquinolone resistance among Campylobacter spp. has become a major public health problem [5]. Increases in the proportion of clinical isolates demonstrating ciprofloxacin resistance has been observed in the United Kingdom (UK) and United States (US) [25, 27], while in the European Union (EU) more than half (54.6\%) of humanassociated $C$. jejuni and two-thirds (66.6\%) of C. coli isolates were resistant to ciprofloxacin in 2013 [28].

Australia has previously reported low rates of fluoroquinolone non-susceptibility among clinical Campylobacter isolates (around 2\% in 2006) [29]. This has been credited to a national pharmaceutical subsidy scheme that restricted human quinolone use and through regulation forbidding quinolone use in food-producing animals [30]. More recent studies reveal this situation has changed markedly, with current rates of ciprofloxacin resistance in clinically-derived Campylobacter isolates now ranging between 13 and 20\% [31,32].

Overseas travel is a well-established risk factor for the acquisition of ciprofloxacin-resistant Campylobacter infections [26, 33]. In our study, cases with ciprofloxacin resistance all reported travel to India and South-East Asia, destinations associated with high rates of antimicrobial resistance among enteric pathogens (including Campylobacter spp.) [34, 35]. Nevertheless, the majority of ciprofloxacin non-susceptible isolates in our study had no recent overseas travel identified, meaning factors associated with domestic acquisition of ciprofloxacin resistance require greater consideration. US data similarly shows increases in domestically acquired ciprofloxacin resistance [27].

We observed that ciprofloxacin non-susceptibility was also associated with previous hospitalisation with campylobacteriosis. There is a paucity of population-level data on recurrent hospitalisations involving non-susceptible Campylobacter isolates. However, rates of recurrent campylobacteriosis in community settings have been reported to be as high as 248 episodes per 100,000 cases per year in the five years following an initial infection [36]. Explanations for our finding could be either host or pathogen-related, including higher rates of humoral immunodeficiency in patients hospitalised with recurrent campylobacteriosis $[11,37]$ or because of de novo mutations or increases in resistant organisms already present at subclinical levels.

While there has been debate around isolate nonsusceptibility and disease severity, reanalysis of the issue appears to show no substantial clinical differences between resistant and susceptible Campylobacter isolates [38]. Consequently, our finding of reduced odds for bloody diarrhoea and tachycardia among admissions with ciprofloxacin non-susceptibility and any antimicrobial non-susceptibility respectively, most likely represents the so-called "healthy traveller" effect [39]. 


\section{Factors associated with antibiotic treatment} during admission

During the study period we observed statistically significant evidence of an increase in the proportion of Campylobacter-associated hospitalisations receiving antibiotic treatment. Antimicrobial therapy for Campylobacter enterocolitis is not routinely advised but may be recommended for patients with or at risk of severe disease, including high volume or bloody diarrhoea, high fever, symptom duration greater than one week, pregnancy or immunocompromised status $[2,10,40]$. Given that hospitalisation can be viewed as a marker of disease severity [41], the rates of treatment within our study population might be expected to differ from those among non-hospitalised campylobacteriosis cases.

Other research on campylobacteriosis in the ACT has found no concomitant increase in hospitalisations during the same period as the current study [6]. Antibiotic treatment rates may be a reflection of local treatment practices rather than a response to disease severity, with data showing rates of appropriate prescribing and compliance with antibiotic guidelines in ACT hospitals to be the lowest in Australia during the study period [42].

One-third of Campylobacter-associated hospitalisations received antibiotics, either empirically or as targeted therapy for confirmed campylobacteriosis. Second generation fluoroquinolones-mainly per oral ciprofloxacin-comprised $80 \%$ of treatment, with macrolides the remainder. Australia has been successful in efforts to limit use of quinolones in humans and to prohibit their use in food-producing animals [30]. This has preserved their clinical use in Australia, with ciprofloxacin and norfloxacin remaining as empirical treatment options for acute infectious diarrhoea, while being recommended alongside azithromycin for treatment of domestically acquired Campylobacter enteritis [40]. Conversely, the high rates of quinolone resistance experienced in the UK, EU and US has seen macrolide treatment recommended or a greater emphasis placed on travel history and knowledge of local resistance patterns to guide empirical prescribing $[43,44]$.

Within our hospitalised study population, the strongest predictor of antibiotic treatment was collection of a blood specimen for culture. Both antibiotic prescription and ordering of blood cultures are clinical decisions, suggesting that the underlying clinical context observed by treating clinicians impacts both practices inducing them to be positively associated. Admission under specific clinical units, including Gastroenterology, Infectious Diseases and General Medicine were also associated with increased odds of receiving antimicrobial therapy. Hospitalisation implies a higher level of morbidity, potentially explaining the higher likelihood of antibiotic administration. Variation in treatment focus could also be expected between subspecialties, especially when underlying comorbidities are exacerbated. Such decision making may be further influenced by routine clinical behaviour, unease regarding the consequences of BSI or by the acceptability of not obtaining blood cultures among particular specialities [4].

Age was also found to be an important factor in treatment, with paediatric admissions being less likely to receive antibiotics. This finding reflects that fluoroquinolones-the most commonly prescribed antibiotic class- are not recommended for use in children due to safety concerns [45]. We also observed that patients aged 40-49 years were more likely to be prescribed antibiotics. Reasons for this are less certain, but around $20 \%$ of admissions to high prescribing units such as Gastroenterology and Infectious Diseases were in this age range.

Vomiting and electrolyte imbalance were also associated with provision of antibiotic therapy. Vomiting is a less frequently reported symptom of campylobacteriosis but serves as an indicator for disease severity [46] and a predictor of bacteraemia [47]. While we did not assess the severity of dehydration, it is likely that a population such as ours included a higher proportion of cases with more pronounced symptomatology and severity of symptoms compared with non-hospitalised cases.

\section{Limitations}

There are a number of potential limitations with our study. Firstly, our rates of bacteraemia may underestimate the true incidence, as we identified cases using only public hospital laboratory data. Other cases of Campylobacter bacteraemia may have been diagnosed and managed in the community or the private hospital sector, although the clinical significance of these is less certain. A second limitation relates to the precision of our model estimates, with the small numbers of observations for some outcomes making detection of clinically meaningful associations challenging. Despite this, the observed associations still align plausibly with Campylobacter's epidemiology. A third limitation relates to the generalisation of our findings. Our study population was hospital-based and drawn from a single Australian territory, with regional and international differences in the epidemiology of campylobacteriosis being observed in high income settings $[14,22,48]$. Finally, antimicrobial susceptibility testing and bacterial speciation were not performed on all isolates, limiting exploration of species-specific features such as higher macrolide resistance rates among C. coli isolates [49]. 


\section{Conclusions}

Campylobacter infections cause a substantial disease burden, as reflected by the high number of hospitalisations and high incidence of bacteraemia in our study. While a spectrum of illness can be observed among hospitalisations, many cases exhibit signs suggestive of systemic disease. Furthermore, both the proportion of cases receiving antibiotic treatment and those having isolates that were non-susceptible to standard antimicrobials increased over time. Given the increasing incidence of Campylobacter infections, particularly among older patients, understanding hospitalisation burden becomes increasingly important. This study provides some evidence in relation to clinical factors influencing the management of hospitalised cases in high income settings.

\section{Abbreviations}

ACT: Australian Capital Territory; aOR: Adjusted Odds Ratio; BSI: Blood Stream Infection; Cl: Confidence Interval; EU: European Union; ICD: International Classification of Diseases; UK: United Kingdom; US: United States.

\section{Acknowledgements \\ We would like to thank the following: ACT Pathology for provision of data on public hospital-generated Campylobacter isolates; ACT Health Protection Service for provision of surveillance data for campylobacteriosis in the ACT; hospital data services staff provision of administrative data for Campylobacter- associated hospitalisations and medical records staff for facilitating access to case medical records identified via morbidity coding and/or data linkage.}

\section{Authors' contributions}

CM planned the study, undertook data collection, performed analysis and drafted the manuscript. BO provided specific advice on analyses. CM, KK, BO, LS and MK all contributed to and approved the final manuscript. All authors read and approved the final manuscript.

\section{Funding}

The National Health and Medical Research Council provided funding to authors CM [APP107490] and MK [APP1145997]. The funding body had no role in the design of the study, collection, analysis and interpretation of data or in writing of the manuscript.

\section{Availability of data and materials}

The data that support the findings of this study are available from the ACT Government Health Directorate and Calvary Health Care (Bruce). Restrictions apply to the availability of these data, which were used under approvals for the current study and so are not publicly available. Data are however available from the authors upon reasonable request and with permission of the ACT Government Health Directorate and Calvary Health Care (Bruce).

\section{Declarations}

\section{Ethics approval and consent to participate}

Ethics approvals were obtained from the Human Research Ethics Committee of the ACT Government Health Directorate (Ref. ETHLR.14.121), Calvary Health Care (Bruce) (Ref. (22-2014) and the Australian National University (Ref. 2014/223). These approvals included permission to access inpatient medical records subject to the study data set being anonymised before use. Individual patient consent was not required.

\section{Consent for publication}

Not applicable.

\section{Competing interests}

The authors declare they have no competing interests.

\section{Author details}

${ }^{1}$ National Centre for Epidemiology and Population Health, Research School of Population Health, Australian National University, 2602 Canberra, ACT, Australia. ${ }^{2}$ Department of Microbiology, Canberra Hospital and Health Services, Canberra, ACT, Australia. ${ }^{3}$ School of Public Health, University of Queensland, Brisbane, QLD, Australia.

Received: 22 September 2020 Accepted: 10 August 2021

Published online: 21 August 2021

\section{References}

1. Kaakoush NO, et al. Global epidemiology of Campylobacter infection. Clin Microbiol Rev. 2015;28(3):687-720.

2. Zollner-Schwetz I, Krause R. Therapy of acute gastroenteritis: role of antibiotics. Clin Microbiol Infect. 2015;21(8):744-9.

3. Nielsen $\mathrm{H}$, et al. Bacteraemia as a result of Campylobacter species: a population-based study of epidemiology and clinical risk factors. Clin Microbiol Infect. 2010;16(1):57-61.

4. Linsenmeyer $\mathrm{K}$, et al. Culture if spikes? Indications and yield of blood cultures in hospitalized medical patients. J Hosp Med. 2016;11(5):336-40.

5. Sproston EL, Wimalarathna HML, Sheppard SK. Trends in fluoroquinolone resistance in Campylobacter. Microb Genom. 2018:4(8):1.

6. Moffatt CRM, et al. Campylobacter-associated hospitalisations in an Australian provincial setting. BMC Infect Dis. 2021;21(1):10.

7. Clinical Laboratory Standards Institute (CLSI), Performance standards for antimicrobial susceptibility testing: Twenty-third informational supplement. CLSI document M100-S23. 2013, Clinical and Laboratory Standards Institute Wayne, PA

8. Charlson ME, et al. A new method of classifying prognostic comorbidity in longitudinal studies: development and validation. J Chronic Dis. 1987:40(5):373-83.

9. Australian Bureau of Statistics, Australian Historical Population Statistics; 2014. 2014, Commonwealth of Australia: Canberra.

10. Allos BM. Campylobacter jejuni infections: update on emerging issues and trends. Clin Infect Dis. 2001;32(8):1201-6.

11. Fernandez-Cruz A, et al. Campylobacter bacteremia: clinical characteristics, incidence, and outcome over 23 years. Med (Baltim) 2010;89(5):319-30.

12. Schonheyder HC, Sogaard P, Frederiksen W. A survey of Campylobacter bacteremia in three Danish counties, 1989 to 1994. Scand J Infect Dis. 1995;27(2):145-8.

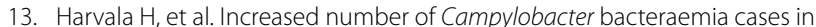
Sweden, 2014. Clin Microbiol Infect. 2016;22(4):391-3.

14. Moffatt $C R$, et al. The campylobacteriosis conundrum - examining the incidence of infection with Campylobacter sp. in Australia, 1998-2013. Epidemiol Infect. 2017;145(4):839-47.

15. Coburn B, et al. Does this adult patient with suspected bacteremia require blood cultures? JAMA. 2012;308(5):502-11.

16. Jones GR, Lowes JA. The systemic inflammatory response syndrome as a predictor of bacteraemia and outcome from sepsis. QJM. 1996:89(7):515-22.

17. James MT, et al. Risk of bloodstream infection in patients with chronic kidney disease not treated with dialysis. Arch Intern Med. 2008;168(21):2333-9.

18. Foley RN. Infections in patients with chronic kidney disease. Infect Dis Clin North Am. 2007;21(3):659-72.

19. Eliakim-Raz N, Bates DW, Leibovici L. Predicting bacteraemia in validated models-a systematic review. Clin Microbiol Infect. 2015;21(4):295-301.

20. Louwen R, et al. Campylobacter bacteremia: a rare and under-reported event? Eur J Microbiol Immunol (Bp). 2012;2(1):76-87.

21. Hussein $\mathrm{K}$, et al. Campylobacter bacteraemia: 16 years of experience in a single centre. Infect Dis (Lond). 2016;48(11-12):796-9.

22. Pacanowski J, et al. Campylobacter bacteremia: clinical features and factors associated with fatal outcome. Clin Infect Dis. 2008;47(6):790-6.

23. Feodoroff $\mathrm{B}$, et al. Clonal distribution and virulence of Campylobacter jejuni isolates in blood. Emerg Infect Dis. 2013;19(10):1653-5.

24. Pigrau C, et al. Bacteremia due to Campylobacter species: clinical findings and antimicrobial susceptibility patterns. Clin Infect Dis. 1997;25(6):1414-20 
25. Cody AJ, et al. Ciprofloxacin-resistant campylobacteriosis in the UK. Lancet. 2010; 376(9757):1987.

26. Post A, et al. Antibiotic susceptibility profiles among Campylobacter isolates obtained from international travelers between 2007 and 2014. Eur J Clin Microbiol Infect Dis. 2017;36(11):2101-7.

27. Geissler AL, et al. Increasing Campylobacter infections, outbreaks, and antimicrobial resistance in the United States, 2004-2012. Clin Infect Dis. 2017;65(10):1624-31.

28. European Food Safety Authority (EFSA) and European Centre for Disease Prevention and Control (ECDC). EU Summary Report on antimicrobial resistance in zoonotic and indicator bacteria from humans, animals and food in 2013. EFSA J. 2015;13(2):4036.

29. Unicomb LE, et al. Low-level fluoroquinolone resistance among Campylobacter jejuni isolates in Australia. Clin Infect Dis. 2006;42(10):1368-74.

30. Cheng AC, et al. Control of fluoroquinolone resistance through successful regulation, Australia. Emerg Infect Dis. 2012;18(9):1453-60.

31. Devi A, et al. Antimicrobial susceptibility of clinical isolates of Campylobacter jejuni from New South Wales, Australia. J Glob Antimicrob Resist. 2019;16:76-80.

32. Wallace R, et al., Status of antimicrobial resistance in clinical isolates of Campylobacter jejuni and Campylobacter jejuni in Australia. J Clin Microbiol. 2019.

33. Ricotta EE, et al. Epidemiology and antimicrobial resistance of international travel-associated Campylobacter infections in the United States, 2005-2011. Am J Public Health. 2014;104(7):e108-14.

34. Hoge CW, et al. Trends in antibiotic resistance among diarrheal pathogens isolated in Thailand over 15 years. Clin Infect Dis. 1998;26(2):341-5.

35. Mukherjee P, et al. Campylobacter jejuni in hospitalized patients with diarrhea, Kolkata, India. Emerg Infect Dis. 2013;19(7):1155-6.

36. Arsenault J, et al. Do patients with recurrent episodes of campylobacteriosis differ from those with a single disease event? BMC Public Health. 2011;11:32.

37. Marcotte H, Hammarström L. Immunodeficiencies: significance for gastrointestinal disease, in Viral Gastroenteritis. Elsevier; 2016. pp. 47-71.

38. Wassenaar TM, Kist M, de Jong A. Re-analysis of the risks attributed to ciprofloxacin-resistant Campylobacter jejuni infections. Int J Antimicrob Agents. 2007;30(3):195-201.
39. Ternhag A, et al. Mortality following Campylobacter infection: a registrybased linkage study. BMC Infect Dis. 2005;5:70.

40. Acute infectious diarrhoea. In Electronic Therapeutic Guidelines Complete. 2019. https://tgldcdp.tg.org.au/viewTopic?topicfile=acute-gastr oenteritis.

41. Helms M, Simonsen J, Molbak K. Foodborne bacterial infection and hospitalization: a registry-based study. Clin Infect Dis. 2006;42(4):498-506.

42. Turnidge JD, et al. Antimicrobial use in Australian hospitals: how much and how appropriate? Med J Aust. 2016;205(10):S16-20.

43. National Institute for Health Care Excellence (NICE) and Public Health England (PHE), Summary of antimicrobial prescribing guidance-managing common infections; 2019.

44. Shane AL, et al. 2017 Infectious Diseases Society of America Clinical Practice Guidelines for the Diagnosis and Management of Infectious Diarrhea. Clin Infect Dis. 2017;65(12):1963-73.

45. Patel K, Goldman JL. Safety concerns surrounding quinolone use in children. J Clin Pharmacol. 2016;56(9):1060-75.

46. Gillespie IA, et al. Investigating vomiting and/or bloody diarrhoea in Campylobacter jejuni infection. J Med Microbiol. 2006;55(Pt 6):741-6.

47. Shapiro $\mathrm{NI}$, et al. Who needs a blood culture? A prospectively derived and validated prediction rule. J Emerg Med. 2008;35(3):255-64.

48. Feodoroff B, et al. A nationwide study of Campylobacter jejuni and Campylobacter coli bacteremia in Finland over a 10-year period, 1998-2007, with special reference to clinical characteristics and antimicrobial susceptibility. Clin Infect Dis. 2011;53(8):e99-106.

49. Bolinger $\mathrm{H}$, Kathariou $\mathrm{S}$. The current state of macrolide resistance in Campylobacter spp.: trends and impacts of resistance mechanisms. Appl Environ Microbiol. 2017;83(12):e00416.

\section{Publisher's Note}

Springer Nature remains neutral with regard to jurisdictional claims in published maps and institutional affiliations.
Ready to submit your research? Choose BMC and benefit from:

- fast, convenient online submission

- thorough peer review by experienced researchers in your field

- rapid publication on acceptance

- support for research data, including large and complex data types

- gold Open Access which fosters wider collaboration and increased citations

- maximum visibility for your research: over 100M website views per year

At BMC, research is always in progress.

Learn more biomedcentral.com/submissions 